Commun. Korean Math. Soc. 28 (2013), No. 1, pp. 191-207

http://dx.doi.org/10.4134/CKMS.2013.28.1.191

\title{
THE SHRINKING PROJECTION METHODS FOR HEMI-RELATIVELY NONEXPANSIVE MAPPINGS, VARIATIONAL INEQUALITIES AND EQUILIBRIUM PROBLEMS
}

\author{
Zi-Ming Wang, Mi Kwang Kang, and Yeol Je Cho
}

\begin{abstract}
In this paper, we introduce the shrinking projection method for hemi-relatively nonexpansive mappings to find a common solution of variational inequality problems and equilibrium problems in uniformly convex and uniformly smooth Banach spaces and prove some strong convergence theorems to the common solution by using the proposed method.
\end{abstract}

\section{Introduction}

Let $E$ be a Banach space and $E^{*}$ the dual space of $E$. Let $C$ be a nonempty closed convex subset of $E$. Let $J$ be the normalized duality mapping from $E$ into $2^{E^{*}}$ defined by

$$
J x=\left\{f \in E^{*}:\langle f, x\rangle=\|x\|^{2}=\|f\|^{2}\right\}, \quad \forall x \in E,
$$

where $\langle\cdot, \cdot\rangle$ denotes the generalized duality pairing.

It is known that the duality mapping $J$ has the following properties:

(1) If $E$ is smooth, then $J$ is single-valued;

(2) If $E$ is strictly convex, then $J$ is one-to-one;

(3) If $E$ is reflexive, then $J$ is surjective;

(4) If $E$ is uniformly smooth, then $J$ is uniformly norm-to-norm continuous on each bounded subset of $E$;

(5) If $E^{*}$ is uniformly convex, then $J$ is uniformly continuous on bounded subsets of $E$ and $J$ is singe-valued and also one-to-one; see [1-4, 17, 19].

Received February 14, 2012.

2010 Mathematics Subject Classification. 47H05, 47H09, 47J25.

Key words and phrases. variational inequality, equilibrium problem, hemi-relatively nonexpansive mapping, shrinking projection method.

The third author was supported by the Korea Research Foundation Grant funded by the Korean Government (KRF-2011-0021821). 
As is well-known to all, variational inequalities are being used as a mathematical programming tool in modeling a wide class of problems arising in several branches of pure and applied sciences; for example, operations research, economic equilibrium and engineering design.

In this paper, we consider the following variational inequality: Find $x \in C$ such that

$$
\langle A x, y-x\rangle \geq 0, \quad \forall y \in C
$$

A point $x_{0} \in C$ is called a solution of the variational inequality (1.1) if $\left\langle A x_{0}, y-x_{0}\right\rangle \geq 0$. The solutions set of the variational inequality (1.1) is denoted by $V I(A, C)$. When $A$ is provided with some monotonicity, many iterative methods for solving the variational inequality (1.1) have been developed; see $[5-13]$.

Most recently, utilizing shrinking projection method, Ying Liu [11] established the following strong convergence theorem for relatively weak nonexpansive mapping and variational inequality in a uniformly convex and uniformly smooth Bananch space.

Theorem 1.1 ([11, Theorem 3.1]). Let $E$ be a uniformly convex and uniformly smooth Banach space and $C$ be a nonempty closed convex subset of $E$. Assume that $A$ is a continuous operator of $C$ into $E^{*}$ satisfying the conditions (1.2) and (1.3) and $S: C \rightarrow C$ is a relatively weak nonexpansive mapping with $F:=F(S) \cap V I(A, C) \neq \emptyset$. Then the sequence $\left\{x_{n}\right\}$ generated by the following iterative scheme:

$$
\left\{\begin{array}{l}
x_{0} \in C \text { chosen arbitrarily, } \\
z_{n}=\Pi_{C}\left(\alpha_{n} J x_{n}+\left(1-\alpha_{n}\right) J S x_{n}\right), \\
y_{n}=J^{-1}\left(\delta_{n} J x_{n}+\left(1-\delta_{n}\right) J \Pi_{C}\left(J z_{n}-\beta A z_{n}\right)\right), \\
C_{0}=\left\{z \in C: \phi\left(z, y_{0}\right) \leq \phi\left(z, x_{0}\right)\right\}, \\
C_{n}=\left\{z \in C_{n-1} \cap Q_{n-1}: \phi\left(z, y_{n}\right) \leq \phi\left(z, x_{n}\right)\right\}, \\
Q_{0}=C, \\
Q_{n}=\left\{z \in C_{n-1} \cap Q_{n-1}:\left\langle J x_{0}-J x_{n}, x_{n}-z\right\rangle \geq 0\right\}, \\
x_{n+1}=\Pi_{C_{n} \cap Q_{n}} J x_{0}, \quad \forall n \geq 1,
\end{array}\right.
$$

where the sequences $\left\{\alpha_{n}\right\}$ and $\left\{\delta_{n}\right\}$ satisfy the following conditions:

$$
0 \leq \delta_{n}<1, \quad \limsup _{n \rightarrow \infty} \delta<1, \quad 0<\alpha_{n}<1, \quad \liminf _{n \rightarrow \infty} \alpha_{n}(1-\alpha)>0,
$$

converges strongly to $\Pi_{F(S) \cap V I(A, C)} J x_{0}$.

Here, we remark that Theorem 1.1 improved the relevant result of Jinlu Li [10] and Jianghua Fan [7], in detail that Theorem 1.1 removed the compactness of $J-\beta A$ instead by the continuity of $A$, and obtained a strong convergence result. 
Recall that a mapping $A: D(A) \subset E \rightarrow E^{*}$ is said to be monotone if the following inequality holds:

$$
\langle A x-A y, x-y\rangle \geq 0, \quad \forall x, y \in D(A) .
$$

$A$ is said to be $\lambda$-inverse strongly monotone if there exists a positive real number $\lambda$ such that

$$
\langle A x-A y, x-y\rangle \geq \lambda\|A x-A y\|^{2}, \quad \forall x, y \in D(A) .
$$

If $A$ is $\lambda$-inverse strongly monotone, then it is Lipschitz continuous with constant $\frac{1}{\lambda}$, i.e., $\|A x-A y\| \leq \frac{1}{\lambda}\|x-y\|, \forall x, y \in D(A)$, and hence uniformly continuous.

For finding an element of a nonexpansive mapping and $V I(A, C)$, Takahashi and Toyoda [20] introduced the following iterative scheme in a Hilbert space $H$ :

$$
x_{n+1}=\alpha_{n} x_{n}+\left(1-\alpha_{n}\right) S P_{C}\left(x_{n}-\mu_{n} A x_{n}\right), \quad \forall n \geq 1,
$$

where $x_{0} \in C, P_{C}$ is a metric projection of $H$ onto $C, A$ is a $\lambda$-inverse strongly monotone operator. Furthermore they proved a weak convergence theorem:

Theorem 1.2 ([20, Theorem 3.1]). Let $C$ be a closed convex subset of a real Hilbert space $H$. Let $\lambda>0$. Let $A$ be an $\lambda$-inverse strongly-monotone mapping of $C$ into $H$, and let $S$ be a nonexpansive mapping of $C$ into itself such that $F(S) \cap V I(A, C) \neq \emptyset$. Let $\left\{x_{n}\right\}$ be a sequence generated by (1.5) for all $n \in$ $\mathbb{N} \cup\{0\}$, where $\left\{\mu_{n}\right\} \subset[a, b]$ for some $a, b \in(0,2 \lambda)$ and $\left\{\alpha_{n}\right\} \subset[c, d]$ for some $c, d \in(0,1)$. Then $\left\{x_{n}\right\}$ converges weakly to $z \in F(S) \cap V I(A, C)$, where $z=\lim _{n \rightarrow \infty} P_{F(S) \cap V I(A, C)} x_{n}$.

On the other hand, the equilibrium problem introduced in [3] in 1994, is always a hot topic of intensive research efforts, because it has a great impact and influence in the development of several branches of pure and applied sciences. It has been shown that equilibrium problem theory provided a novel and unified treatment of a wide class of problems which arise in economics, finance, physics, image reconstruction, ecology, transportation, network, elasticity and optimization. Numerous problems in physics, optimization and economics reduce to finding a solution of equilibrium problem. Some methods have been proposed to solve the equilibrium problem; see [10-13].

Let $f: C \times C \rightarrow \mathbb{R}$ be a bifunction. The equilibrium problem for $f$ is as follows: Find $\hat{x} \in C$ such that

$$
f(\hat{x}, y) \geq 0, \quad \forall y \in C .
$$

The set of solutions of the problem (1.6) is denoted by $E P(f)$. For solving the equilibrium problem, ones always assume that a bifunction $f$ satisfies the following conditions:

(A1) $f(x, x)=0$ for all $x \in C$;

(A2) $f$ is monotone, that is, $f(x, y)+f(y, x) \leq 0$ for all $x, y \in C$; 
(A3) For all $x, y, z \in C$,

$$
\limsup _{t \downarrow 0} f(t z+(1-t) x, y) \leq f(x, y) ;
$$

(A4) For all $x \in C, f(x, \cdot)$ is convex and lower semicontinuous.

For example, let $A$ be a continuous and monotone operator of $C$ into $E^{*}$ and define

$$
f(x, y)=\langle A x, y-x\rangle, \quad \forall x, y \in C .
$$

Then $f$ satisfies (A1)-(A4).

In this paper, motivated and inspired by the results mentioned above, we introduce a new hybrid projection algorithm based on the shrinking projection method for a closed hemi-relatively nonexpansive mapping, variational inequality, equilibrium problem. Using the new algorithm, we prove some strong convergence theorem which approximate a common element in the fixed points set of the closed hemi-relatively nonexpansive mappings, the solutions set of a variational inequality and the solutions set of the equilibrium problem in a uniformly convex and uniformly smooth Banach space. Our results extend and improve the recent ones announced by Li [10], Fan [7], Liu [11], Takahashi and Toyoda [3] and many others.

\section{Preliminaries}

A Banach space $E$ is said to be strictly convex if $\frac{x+y}{2}<1$ for all $x, y \in$ $E$ with $\|x\|=\|y\|=1$ and $x \neq y$. It is said to be uniformly convex if $\lim _{n \rightarrow \infty}\left\|x_{n}-y_{n}\right\|=0$ for any two sequences $\left\{x_{n}\right\}$ and $\left\{y_{n}\right\}$ in $E$ such that $\left\|x_{n}\right\|=\left\|y_{n}\right\|=1$ and $\lim _{\rightarrow \infty}\left\|\frac{x_{n}+y_{n}}{2}\right\|=1$.

Let $U_{E}=\{x \in E:\|x\|=1\}$ be the unit sphere of $E$. Then the Banach space $E$ is said to be smooth provided

$$
\lim _{t \rightarrow 0} \frac{\|x+t y\|-\|x\|}{t}
$$

exists for each $x, y \in U_{E}$. It is also said to be uniformly smooth if the limit (2.1) is attained uniformly for $x, y \in U_{E}$.

It is well known that, if $E$ is uniformly smooth, then $J$ is uniformly normto-norm continuous on each bounded subset of $E$ and, if $E$ is uniformly smooth if and only if $E^{*}$ is uniformly convex.

Let $C$ be a closed convex subset of $E$ and $T$ be a mapping from $C$ into itself. A point $p$ in $C$ is said to be an asymptotic fixed point of $T$ if $C$ contains a sequence $\left\{x_{n}\right\}$ which converges weakly to $p$ such that the strong $\lim _{n \rightarrow \infty}\left(x_{n}-\right.$ $\left.T x_{n}\right)=0$. The set of asymptotic fixed points of $T$ is denoted by $\widehat{F}(T)$.

Recall that an operator $T$ in Banach space $E$ is said to be closed if $x_{n} \rightarrow x$ and $T x_{n} \rightarrow y$ implies $T x=y$.

A mapping $T$ from $C$ into itself is said to be nonexpansive if

$$
\|T x-T y\| \leq\|x-y\|, \quad \forall x, y \in C .
$$


The mapping $T$ is said to be relatively nonexpansive [14-16] if

$$
\widehat{F}(T)=F(T) \neq \emptyset, \quad \phi(p, T x) \leq \phi(p, x), \quad \forall x \in C, p \in F(T) .
$$

The asymptotic behavior of a relatively nonexpansive mapping was studied in [14-16]. A point $p \in C$ is called a strong asymptotic fixed point of $T$ if $C$ contains a sequence $\left\{x_{n}\right\}$ which converges strongly to $p$ such that

$$
\lim _{n \rightarrow \infty}\left(x_{n}-T x_{n}\right)=0 .
$$

The set of strong asymptotic fixed points of $T$ is denoted by $\widetilde{F}(T)$.

A mapping $T$ from $C$ into itself is said to be relatively weak nonexpansive if

$$
\widetilde{F}(T)=F(T) \neq \emptyset, \quad \phi(p, T x) \leq \phi(p, x), \quad \forall x \in C, p \in F(T) .
$$

The mapping $T$ is said to be hemi-relatively nonexpansive if

$$
F(T) \neq \emptyset, \quad \phi(p, T x) \leq \phi(p, x), \quad \forall x \in C, p \in F(T) .
$$

It is obvious that a relatively nonexpansive mapping is a relatively and weakly nonexpansive mapping and, further, a relatively and weakly nonexpansive mapping are all hemi-relatively nonexpansive, but the converses are not true as in the following example:

Example 2.1 ([18]). Let $E$ be any smooth Banach space and $x_{0} \neq 0$ be any element of $E$. We define a mapping $T: E \rightarrow E$ as follows: For all $n \geq 1$,

$$
T(x)= \begin{cases}\left(\frac{1}{2}+\frac{1}{2^{n+1}}\right) x_{0}, & \text { if } x=\left(\frac{1}{2}+\frac{1}{2^{n}}\right) x_{0}, \\ -x, & \text { if } x \neq\left(\frac{1}{2}+\frac{1}{2^{n}}\right) x_{0} .\end{cases}
$$

Then $T$ is a hemi-relatively nonexpansive mapping, but it is not relatively nonexpansive mapping.

Next, we give an important example which is also hemi-relatively nonexpansive.

Example 2.2 ([15]). Let $E$ be a strictly convex reflexive smooth Banach space. Let $A$ be a maximal monotone operator of $E$ into $E^{*}$ and $J_{r}$ be the resolvent for $A$ with $r>0$. Then $J_{r}=(J+r A)^{-1} J$ is a hemi-relatively nonexpansive mapping from $E$ onto $D(A)$ with $F\left(J_{r}\right)=A^{-1} 0$.

In $[8,2]$, Alber introduced the functional $V: E^{*} \times E \rightarrow \mathbb{R}$ defined by

$$
V(\phi, x)=\|\phi\|^{2}-2\langle\phi, x\rangle+\|x\|^{2},
$$

where $\phi \in E^{*}$ and $x \in E$. It is easy to see that

$$
V(\phi, x) \geq(\|\phi\|-\|x\|)^{2}
$$

and so the functional $V: E^{*} \times E \rightarrow \mathbb{R}^{+}$is nonnegative.

In order to prove our results in the next section, we present several necessary definitions and lemmas. 
Definition 2.3 ([9]). If $E$ be a uniformly convex and uniformly smooth Banach space, then the generalized projection $\Pi_{C}: E^{*} \rightarrow C$ is a mapping that assigns an arbitrary point $\phi \in E^{*}$ to the minimum point of the functional $V(\phi, x)$, i.e., a solution to the minimization problem

$$
V\left(\phi, \Pi_{C}(\phi)\right)=\inf _{y \in C} V(\phi, y) .
$$

Li [10] proved that the generalized projection operator $\Pi_{C}: E^{*} \rightarrow C$ is continuous if $E$ is a reflexive, strictly convex and smooth Banach space.

Consider the function $\phi: E \times E \rightarrow \mathbb{R}$ is defined by

$$
\phi(x, y)=V(J y, x), \quad \forall x, y \in E .
$$

The following properties of the operator $\Pi_{C}$ and $V$ are useful for our paper; see, for example, $[1,10]$.

(B1) $V: E^{*} \times E \rightarrow \mathbb{R}$ is continuous;

(B2) $V(\phi, x)=0$ if and only if $\phi=J x$;

(B3) $V\left(J \Pi_{C}(\phi), x\right) \leq V(\phi, x)$ for all $\phi \in E^{*}$ and $x \in E$;

(B4) The operator $\Pi_{C}$ is $J$ fixed at each point $x \in E^{*}$ and $x \in E$;

(B5) If $E$ is smooth, then, for any given $\phi \in E^{*}$ and $x \in C, x \in \Pi_{C}(\phi)$ if and only if

$$
\langle\phi-J x, x-y\rangle \geq 0, \quad \forall y \in C ;
$$

(B6) The operator $\Pi_{C}: E^{*} \rightarrow c$ is single valued if and only if $E$ is strictly convex;

(B7) If $E$ is smooth, then, for any given point $\phi \in E^{*}$ and $x \in \Pi_{C}(\phi)$, the following inequality holds:

$$
V(J x, y) \leq V(\phi, y)-V(\phi, x), \quad \forall y \in C ;
$$

(B8) $v(\phi, X)$ is convex with respect to $\phi$ when $x$ is fixed and with respect to $x$ when $\phi$ is fixed;

(B9) If $E$ is reflexive, then, for any point $\phi \in E^{*}, \Pi_{C}(\phi)$ is a nonempty closed convex and bounded subset of $C$.

Using some properties of the generalized projection operator $\Pi_{C}$, Alber [1] proved the following theorem:

Lemma 2.4 ([1]). Let $E$ be a strictly convex reflexive smooth Banach space. Let $A$ be an arbitrary operator from a Banach space $E$ to $E^{*}$ and $\beta$ be an arbitrary fixed positive number. Then $x \in C \subset E$ is a solution of the variational inequality (1.1) if and only if $x$ is a solution of the following operator equation in $E$ :

$$
x=\Pi_{C}(J x-\beta A x) .
$$

Lemma 2.5 ([9]). Let $E$ be a uniformly convex smooth Banach space and $\left\{y_{n}\right\},\left\{z_{n}\right\}$ be two sequences of $E$ such that either $\left\{y_{n}\right\}$ or $\left\{z_{n}\right\}$ is bounded. If $\lim _{n \rightarrow \infty} \phi\left(y_{n}, z_{n}\right)=0$, then $\lim _{n \rightarrow \infty}\left\|y_{n}-z_{n}\right\|=0$. 
Lemma 2.6 ([5]). Let $E$ be a uniformly convex and uniformly smooth Banach space. We have

$$
\|\phi+\Phi\|^{2} \leq\|\phi\|^{2}+2\langle\Phi, J(\phi+\Phi)\rangle, \quad \forall \phi, \Phi \in E^{*} .
$$

From Qin et al. [16], the following lemma can be obtained immediately.

Lemma 2.7. Let $E$ be a uniformly convex Banach space, $s>0$ be a positive number and $B_{s}(0)$ be a closed ball of $E$. Then there exists a continuous, strictly increasing and convex function $g:[0, \infty) \rightarrow[0, \infty)$ with $g(0)=0$ such that

$$
\left\|\Sigma_{i=1}^{N}\left(\alpha_{i} x_{i}\right)\right\|^{2} \leq \Sigma_{i=1}^{N}\left(\alpha_{i}\left\|x_{i}\right\|^{2}\right)-\alpha_{i} \alpha_{j} g\left(\left\|x_{i}-x_{j}\right\|\right)
$$

for all $x_{1}, x_{2}, \ldots, x_{N} \in B_{s}(0)=\{x \in E:\|x\| \leq s\}, i \neq j$ for all $i, j \in$ $\{1,2, \ldots, N\}$ and $\alpha_{1}, \alpha_{2}, \ldots, \alpha_{N} \in[0,1]$ such that $\sum_{i=1}^{N} \alpha_{i}=1$.

Lemma 2.8 ([3]). Let $C$ be a closed and convex subset of a smooth, strictly convex and reflexive Banach spaces $E, f$ be a bifunction from $C \times C$ to $\mathbb{R}$ satisfying the conditions (B1)-(B4) and let $r>0, x \in E$. Then there exists $z \in C$ such that

$$
f(z, y)+\frac{1}{r}\langle J z-J x, y-z\rangle \geq 0, \quad \forall y \in C .
$$

Lemma 2.9 ([21]). Let $C$ be a closed and convex subset of a uniformly smooth, strictly convex and reflexive Banach spaces $E$, let $f$ be a bifunction from $C \times C$ to $\mathbb{R}$ satisfying (B1)-(B4). For all $r>0$ and $x \in E$, define the mapping

$$
T_{r} x=\left\{z \in C: f(z, y)+\frac{1}{r}\langle J z-J x, y-z\rangle \geq 0, \forall y \in C\right\} .
$$

Then the following hold:

(C1) $T_{r}$ is single-valued;

(C2) $T_{r}$ is a firmly nonexpansive-type mapping, that is, for all $x, y \in E$,

$$
\left\langle J T_{r} x-J T_{r} y, T_{r} x-T_{r} y\right\rangle \leq\left\langle J x-J y, T_{r} x-T_{r} y\right\rangle ;
$$

(C3) $F\left(T_{r}\right)=\hat{F}\left(T_{r}\right)=E P(f)$;

(C4) $E P(f)$ is closed and convex.

Lemma 2.10 ([21]). Let $C$ be a closed convex subset of a smooth, strictly convex, and reflexive Banach space $E$, let $f$ be a bifunction from $C \times C$ to $\mathbb{R}$ satisfying (C1)-(C4) and let $r>0$. Then, for all $x \in E$ and $q \in F\left(T_{r}\right)$,

$$
\phi\left(q, T_{r} x\right)+\phi\left(T_{r} x, x\right) \leq \phi(q, x) .
$$

Lemma 2.11 ([11, Lemma 2.5]). Let $E$ be a uniformly convex and uniformly smooth Banach space and let $C$ be a nonempty closed and convex subset of $E$. Suppose that there exists a positive number $\eta$ such that

$$
\left\langle A x, J^{-1}(J x-\eta A x)\right\rangle \geq 0, \quad \forall x \in C,
$$

and

$$
\langle A x, y\rangle \leq 0, \quad \forall x \in K, y \in V I(A, C)
$$


Then $\operatorname{VI}(A, C)$ is closed and convex.

Lemma 2.12 ([11, Lemma 2.6]). If $E$ is a reflexive, strictly convex and smooth Banach space, then $\Pi_{C}=J^{-1}$.

Lemma 2.13 ([11, Lemma 2.6]). Let $E$ be a strictly convex and smooth real Banach space, $C$ be a closed convex subset of $E$ and $T$ be a hemi-relatively nonexpansive mapping from $C$ into itself. Then $F(T)$ is closed and convex.

\section{Main results}

Theorem 3.1. Let $E$ be a uniformly convex and uniformly smooth Banach space and $C$ be a nonempty closed convex subset of $E$. Let $f$ be a bifunction from $C \times C$ to $\mathbb{R}$ satisfying the conditions $\left(\mathrm{A}_{1}\right)-\left(\mathrm{A}_{4}\right)$. Assume that $A$ is a continuous operators of $C$ into $E^{*}$ satisfying the conditions (2.10) and (2.11), and $T: C \rightarrow C$ is a closed hemi-relatively nonexpansive mapping with $\digamma:=$ $F(T) \cap V I(A, C) \cap E P(f) \neq \emptyset$. For an arbitrary element $x_{0} \in C$, put $C_{0}=C$ and let $\left\{x_{n}\right\}$ be a sequence generated by the following iterative scheme:

$$
\left\{\begin{array}{l}
z_{n}=\Pi_{C}\left(J x_{n}-\eta A x_{n}\right), \\
y_{n}=\Pi_{C}\left(\alpha_{n} J x_{0}+\beta_{n} J x_{n}+\gamma_{n} J T z_{n}\right), \\
u_{n} \in C \quad \text { such that } \quad f\left(u_{n}, y\right)+\frac{1}{r_{n}}\left\langle J u_{n}-J y_{n}, y-u_{n}\right\rangle \geq 0, \quad \forall y \in C, \\
C_{n+1}=\left\{z \in C_{n}: \phi\left(z, u_{n}\right) \leq \phi\left(z, y_{n}\right)\right. \\
\quad \leq \alpha_{n} \phi\left(z, x_{0}\right)+\beta_{n} \phi\left(z, x_{n}\right)+\gamma_{n} \phi\left(z, z_{n}\right) \\
\left.\quad \leq \alpha_{n} \phi\left(z, x_{0}\right)+\left(1-\alpha_{n}\right) \phi\left(z, x_{n}\right)\right\} \\
\\
x_{n+1}=\Pi_{C_{n+1}} J x_{0}, \quad \forall n \geq 1,
\end{array}\right.
$$

where $\left\{\alpha_{n}\right\},\left\{\beta_{n}\right\}$ and $\left\{\gamma_{n}\right\}$ are the sequences in $[0,1]$ with the following restrictions:
(a) $\alpha_{n}+\beta_{n}+\gamma_{n}=1$;
(b) $\left\{r_{n}\right\} \subset[a, \infty)$ for some $a>0$;
(c) $\lim _{n \rightarrow \infty} \alpha_{n}=0$ and $\liminf _{n \rightarrow \infty} \beta_{n} \gamma_{n}>0$.

Then the sequence $\left\{x_{n}\right\}$ converges strongly to a point $\Pi_{\digamma} J x_{0}$, where $\Pi_{\digamma}$ is the generalized projection from $C$ onto $\digamma$.

Proof. We divide the proof into five steps.

Step 1. $\Pi_{F} J x_{0}$ and $\Pi_{C_{n+1}} J x_{0}$ are well defined.

From Lemma 2.9(C4), Lemma 2.11 and Lemma 2.13, one has that $\Pi_{F} J x_{0}$ is well defined.

Now, we show that $C_{n}$ is closed and convex for all $n \in \mathbb{N} \cup\{0\}$. From the definitions of $C_{n}$, it is obvious that $C_{n}$ is closed for all $n \in \mathbb{N} \cup\{0\}$.

Next, we prove that $C_{n}$ is convex for all $n \in \mathbb{N} \cup\{0\}$. Since $\phi\left(z, u_{n}\right) \leq \phi\left(z, y_{n}\right)$ is equivalent to

$$
2\left\langle J y_{n}-J u_{n}, z\right\rangle \leq\left\|y_{n}\right\|^{2}-\left\|u_{n}\right\|^{2}
$$


$\phi\left(z, y_{n}\right) \leq \alpha_{n} \phi\left(z, x_{0}\right)+\beta_{n} \phi\left(z, x_{n}\right)+\gamma_{n} \phi\left(w, z_{n}\right)$ is equivalent to

$$
2\left\langle\alpha_{n} J x_{0}+\beta_{n} J x_{n}+\gamma_{n} J z_{n}-J y_{n}, z\right\rangle \leq \alpha_{n}\left\|x_{0}\right\|^{2}+\beta_{n}\left\|x_{n}\right\|^{2}+\gamma_{n}\left\|z_{n}\right\|^{2} ;
$$

and $\left.\alpha_{n} \phi\left(z, x_{0}\right)+\beta_{n} \phi\left(z, x_{n}\right)+\gamma_{n} \phi\left(z, z_{n}\right) \leq \alpha_{n} \phi\left(z, x_{0}\right)+\left(1-\alpha_{n}\right) \phi\left(z, x_{n}\right)\right\}$ is equivalent to

$$
2\left\langle J x_{n}-J z_{n}, z\right\rangle \leq\left\|x_{n}\right\|^{2}-\left\|z_{n}\right\|^{2} .
$$

It follows that $C_{n}$ is convex for all $n \in \mathbb{N} \cup\{0\}$. Thus, for all $n \in \mathbb{N} \cup\{0\}, C_{n}$ is closed and convex and so $\Pi_{C_{n+1}} J x_{0}$ is well defined.

Step 2. $\digamma \subset C_{n}$ for all $n \in \mathbb{N} \cup\{0\}$.

Observe that $\digamma \subset C_{0}=C$ is obvious. Suppose that $\digamma \subset C_{n}$ for some $n \in \mathbb{N}$. Let $w \in \digamma \subset C_{n}$. Then, from the definition of $\phi$ and $V$, the property (B3) of $V$, Lemma 2.6, the conditions (2.10) and (2.11), it follows that

$$
\begin{aligned}
\phi\left(w, \Pi_{C}\left(J x_{n}-\eta A x_{n}\right)\right)= & V\left(J \Pi_{C}\left(J x_{n}-\eta A x_{n}\right), w\right) \\
\leq & V\left(J x_{n}-\eta A x_{n}, w\right) \\
= & \left\|J x_{n}-\eta A x_{n}\right\|^{2}-2\left\langle J x_{n}-\eta A x_{n}, w\right\rangle+\|w\|^{2} \\
\leq & \left\|J x_{n}\right\|^{2}-2 \eta\left\langle A x_{n}, J^{-1}\left(J x_{n}-\eta A x_{n}\right)\right\rangle \\
& -2\left\langle J x_{n}-\eta A z_{n}, w\right\rangle+\|w\|^{2} \\
\leq & \left\|J x_{n}\right\|^{2}-2\left\langle J x_{n}, w\right\rangle+\|w\|^{2} \\
= & \phi\left(w, x_{n}\right) .
\end{aligned}
$$

Since $u_{n}=T_{r_{n}} y_{n}$, applying Lemma 2.10, the properties (B3) and (B8) of the operator $V$ and (3.2), we obtain

$$
\begin{aligned}
\phi\left(w, u_{n}\right) & =\phi\left(w, T_{r_{n}} y_{n}\right) \leq \phi\left(w, y_{n}\right)=V\left(J y_{n}, w\right) \\
& \leq \alpha_{n} V\left(J x_{0}, w\right)+\beta_{n} V\left(J x_{n}, w\right)+\gamma_{n} V\left(J T z_{n}, w\right) \\
& \leq \alpha_{n} \phi\left(w, x_{0}\right)+\beta_{n} \phi\left(w, x_{n}\right)+\gamma_{n} \phi\left(w, z_{n}\right) \\
& \leq \alpha_{n} \phi\left(w, x_{0}\right)+\beta_{n} \phi\left(w, x_{n}\right)+\gamma_{n} \phi\left(w, x_{n}\right) \\
& =\alpha_{n} \phi\left(w, x_{0}\right)+\left(1-\alpha_{n}\right) \phi\left(w, x_{n}\right),
\end{aligned}
$$

which shows that $w \in C_{n+1}$. Depending on the randomicity of $w$, one can learn that $\digamma \subset C_{n}$ for all $n \in \mathbb{N} \cup\{0\}$.

Step 3. $\left\{x_{n}\right\}$ is a Cauchy sequence.

Since $x_{n}=\Pi_{C_{n}} J x_{0}$, and from (2.4), one has

$$
V\left(J x_{0}, x_{n}\right) \leq V\left(J x_{0}, y\right), \quad \forall y \in C,
$$

and $\digamma \subset C_{n} \subset C$, one also has

$$
V\left(J x_{0}, x_{n}\right) \leq V\left(J x_{0}, w\right), \quad \forall w \in \digamma .
$$

Therefore, $\left\{V\left(J x_{0}, x_{n}\right)\right\}$ is bounded. Moreover, from the definition of $V$, it follows that $\left\{x_{n}\right\}$ is bounded. Since $x_{n+1}=\Pi_{C_{n+1}} J x_{0} \in C_{n+1}$ and $x_{n}=$ $\Pi_{C_{n}} J x_{0}$, we have

$$
V\left(J x_{0}, x_{n}\right) \leq V\left(J x_{0}, x_{n+1}\right), \forall n \geq 0 .
$$


Hence $\left\{V\left(J x_{0}, x_{n}\right)\right\}$ is nondecreasing and so $\lim _{n \rightarrow \infty} V\left(J x_{0}, x_{n}\right)$ exists. By the construction of $C_{n}$, we have that $C_{m} \subset C_{n}$ and $x_{m}=\Pi_{C_{m}} J x_{0} \in C_{n}$ for any positive integer $m \geq n$. From the property (B3), we have

$$
V\left(J x_{n}, x_{m}\right) \leq V\left(J x_{0}, x_{m}\right)-V\left(J x_{0}, x_{n}\right)
$$

for all $n \in \mathbb{N} \cup\{0\}$ and any positive integer $m \geq n$. This implies that

$$
V\left(J x_{n}, x_{m}\right) \rightarrow 0 \quad(n, m \rightarrow \infty) .
$$

The definition of $\phi$ implies that

$$
\phi\left(x_{m}, x_{n}\right) \rightarrow 0 \quad(n, m \rightarrow \infty) .
$$

Applying Lemma 2.5, we obtain

$$
\left\|x_{m}-x_{n}\right\| \rightarrow 0 \quad(n, m \rightarrow \infty) .
$$

Hence $\left\{x_{n}\right\}$ is a Cauchy sequence. In view of the completeness of a Banach space $E$ and the closeness of $C$, it follows that

$$
\lim _{n \rightarrow \infty} x_{n}=p
$$

for some $p \in C$.

Step 4. $p \in \digamma$.

First, we show that $p \in F(T)$. In fact, since $x_{n+1} \in C_{n+1}$, we have

$$
\phi\left(x_{n+1}, u_{n}\right) \leq \phi\left(x_{n+1}, x_{n}\right) .
$$

Thus, by (3.4) and Lemma 2.5, we have

$$
\left\|x_{n+1}-u_{n}\right\| \rightarrow 0 \quad(n \rightarrow \infty)
$$

and hence

$$
\left\|x_{n}-u_{n}\right\| \leq\left\|x_{n}-x_{n+1}\right\|+\left\|x_{n+1}-u_{n}\right\| \rightarrow 0 \quad(n \rightarrow \infty),
$$

which implies that

$$
\lim _{n \rightarrow \infty} u_{n}=\lim _{n \rightarrow \infty} x_{n}=p .
$$

On the other hand, since $J$ is uniformly norm-to-norm continuous on bounded sets, one has

$$
\lim _{n \rightarrow \infty}\left\|J x_{n}-J u_{n}\right\|=0 \text {. }
$$

Since $\left\{x_{n}\right\}$ is bounded, $\left\{J x_{n}\right\},\left\{J T x_{n}\right\}$ and $\left\{J S x_{n}\right\}$ are also bounded. Since $E$ is a uniformly smooth Banach space, one knows that $E^{*}$ is a uniformly convex Banach space. Let $r=\sup _{n \geq 0}\left\{\left\|J x_{0}\right\|,\left\|J x_{n}\right\|,\left\|J T x_{n}\right\|\right\}$. Therefore, from Lemma 2.7, it follows that there exists a continuous strictly increasing convex function $g:[0, \infty) \rightarrow[0, \infty)$ satisfying $g(0)=0$ and the inequality $(2.7)$. 
It follows from the property (B3) of the operator $V,(3.2)$ and the definition of $T$ that

$(3.10)$

$$
\begin{aligned}
\phi\left(w, y_{n}\right)= & V\left(J y_{n}, w\right) \\
\leq & V\left(\alpha_{n} J x_{0}+\beta_{n} J x_{n}+\gamma_{n} J T z_{n}, w\right) \\
= & \phi\left(w, J^{-1}\left(\alpha_{n} J x_{0}+\beta_{n} J x_{n}+\gamma_{n} J T z_{n}\right)\right) \\
= & \|w\|^{2}-2 \alpha_{n}\left\langle J x_{0}, w\right\rangle-2 \beta_{n}\left\langle J x_{n}, w\right\rangle-2 \gamma_{n}\left\langle J T z_{n}, w\right\rangle \\
& +\left\|\alpha_{n} J x_{0}+\beta_{n} J x_{n}+\gamma_{n} J T z_{n}\right\|^{2} \\
\leq & \|w\|^{2}-2 \alpha_{n}\left\langle J x_{0}, w\right\rangle-2 \beta_{n}\left\langle J x_{n}, w\right\rangle-2 \gamma_{n}\left\langle J T z_{n}, w\right\rangle \\
& +\alpha_{n}\left\|J x_{0}\right\|^{2}+\beta_{n}\left\|J x_{n}\right\|^{2}+\gamma_{n}\left\|J T z_{n}\right\|^{2}-\beta_{n} \gamma_{n} g\left(\left\|J x_{n}-J T z_{n}\right\|\right) \\
= & \alpha_{n} \phi\left(w, x_{0}\right)+\beta_{n} \phi\left(w, x_{n}\right)+\gamma_{n} \phi\left(w, T z_{n}\right)-\beta_{n} \gamma_{n} g\left(\left\|J x_{n}-J T z_{n}\right\|\right) \\
\leq & \alpha_{n} \phi\left(w, x_{0}\right)+\beta_{n} \phi\left(w, x_{n}\right)+\gamma_{n} \phi\left(w, x_{n}\right)-\beta_{n} \gamma_{n} g\left(\left\|J x_{n}-J T z_{n}\right\|\right) \\
= & \alpha_{n} \phi\left(w, x_{0}\right)+\left(1-\alpha_{n}\right) \phi\left(w, x_{n}\right)-\beta_{n} \gamma_{n} g\left(\left\|J x_{n}-J T z_{n}\right\|\right) .
\end{aligned}
$$

And from (3.3), we get

$$
\phi\left(w, u_{n}\right)=\phi\left(w, T_{r_{n}} y_{n}\right) \leq \phi\left(w, y_{n}\right)
$$

Substituting (3.10) into (3.11), we obtain

$$
\phi\left(w, u_{n}\right) \leq \alpha_{n} \phi\left(w, x_{0}\right)+\left(1-\alpha_{n}\right) \phi\left(w, x_{n}\right)-\beta_{n} \gamma_{n} g\left(\left\|J x_{n}-J T z_{n}\right\|\right) .
$$

The above inequality implies that

(3.12) $\beta_{n} \gamma_{n} g\left(\left\|J x_{n}-J T z_{n}\right\|\right) \leq \alpha_{n} \phi\left(w, x_{0}\right)+\left(1-\alpha_{n}\right) \phi\left(w, x_{n}\right)-\phi\left(w, u_{n}\right)$.

On the other hand, we have

$$
\begin{aligned}
\phi\left(w, x_{n}\right)-\phi\left(w, u_{n}\right) & =2\left\langle J u_{n}-J x_{n}, w\right\rangle+\left\|x_{n}\right\|^{2}-\left\|u_{n}\right\|^{2} \\
& \leq 2\left\langle J u_{n}-J x_{n}, p\right\rangle+\left(\left\|x_{n}\right\|-\left\|u_{n}\right\|\right)\left(\left\|x_{n}\right\|+\left\|u_{n}\right\|\right) \\
& \leq 2\left\|J u_{n}-J x_{n}\right\|\|w\|+\left\|x_{n}-u_{n}\right\|\left(\left\|x_{n}\right\|+\left\|u_{n}\right\|\right) .
\end{aligned}
$$

It follows from (3.7) and (3.9) that

$$
\lim _{n \rightarrow \infty}\left(\phi\left(w, x_{n}\right)-\phi\left(w, u_{n}\right)\right)=0 .
$$

In view of $\lim _{n \rightarrow \infty} \alpha_{n}=0, \liminf _{n \rightarrow \infty} \beta_{n} \gamma_{n}>0$, the inequality (3.12) implies that

$$
g\left(\left\|J x_{n}-J T z_{n}\right\|\right) \rightarrow 0 \quad(n \rightarrow \infty) .
$$

Therefore, from the property of $g$, we get

$$
\left\|J x_{n}-J T z_{n}\right\| \rightarrow 0 \quad(n \rightarrow \infty) .
$$

Furthermore, since $J^{-1}$ is uniformly norm to norm continuous on bounded sets, we see that

$$
\left\|x_{n}-T z_{n}\right\| \rightarrow 0 \quad(n \rightarrow \infty)
$$


On the other hand, by the construction of $C_{n}$, we know that

$$
\begin{aligned}
\phi\left(z, u_{n}\right) \leq \phi\left(z, y_{n}\right) & \leq \alpha_{n} \phi\left(z, x_{0}\right)+\beta_{n} \phi\left(z, x_{n}\right)+\gamma_{n} \phi\left(z, z_{n}\right) \\
& \leq \alpha_{n} \phi\left(z, x_{0}\right)+\left(1-\alpha_{n}\right) \phi\left(z, x_{n}\right) .
\end{aligned}
$$

From $x_{n+1}=\Pi_{C_{n+1}} J x_{0} \in C_{n+1}$, we have

$$
\begin{aligned}
\phi\left(x_{n+1}, u_{n}\right) \leq \phi\left(x_{n+1}, y_{n}\right) & \leq \alpha_{n} \phi\left(x_{n+1}, x_{0}\right)+\beta_{n} \phi\left(x_{n+1}, x_{n}\right)+\gamma_{n} \phi\left(x_{n+1}, z_{n}\right) \\
& \leq \alpha_{n} \phi\left(x_{n+1}, x_{0}\right)+\left(1-\alpha_{n}\right) \phi\left(x_{n+1}, x_{n}\right) .
\end{aligned}
$$

From (3.4) and $\lim _{n \rightarrow \infty} \alpha_{n}=0$, the above inequality implies that

$$
\phi\left(x_{n+1}, z_{n}\right) \rightarrow 0 \quad(n \rightarrow \infty) .
$$

Applying Lemma 2.5, one has

$$
\left\|x_{n+1}-z_{n}\right\| \rightarrow 0 \quad(n \rightarrow \infty)
$$

and, by (3.5), we obtain that

$$
\left\|x_{n}-z_{n}\right\| \leq\left\|x_{n}-x_{n+1}\right\|+\left\|x_{n+1}-z_{n}\right\| \rightarrow 0 \quad(n \rightarrow \infty) .
$$

Thus, from (3.14) and (3.15), one sees that

$$
\left\|z_{n}-T z_{n}\right\| \leq\left\|z_{n}-x_{n}\right\|+\left\|x_{n}-T z_{n}\right\| \rightarrow 0 \quad(n \rightarrow \infty) .
$$

Thus, from the closedness of $T$, we obtain that $p \in F(T)$.

Secondly, we show that $p \in E P(f)$. From $u_{n}=T_{r_{n}} x_{n}$ and the construction of $C_{n}$, one has

$$
\begin{aligned}
\phi\left(u_{n}, y_{n}\right) & =\phi\left(T_{r_{n}} y_{n}, y_{n}\right) \\
& \leq \phi\left(w, y_{n}\right)-\phi\left(w, T_{r_{n}} y_{n}\right) \\
& \leq \phi\left(w, x_{n}\right)-\phi\left(w, T_{r_{n}} y_{n}\right) \\
& \leq \phi\left(w, x_{n}\right)-\phi\left(w, u_{n}\right) .
\end{aligned}
$$

And, by (3.13), it follows that

$$
\phi\left(u_{n}, y_{n}\right) \rightarrow 0 \quad(n \rightarrow \infty) .
$$

Applying Lemma 2.5, we obtain

$$
\left\|u_{n}-y_{n}\right\| \rightarrow 0 \quad(n \rightarrow \infty) .
$$

Since $J$ is a uniformly norm-to-norm continuous on bounded sets, one has

$$
\lim _{n \rightarrow \infty}\left\|J u_{n}-J y_{n}\right\|=0 \text {. }
$$

From the assumption that $r_{n} \geq a$, one has

$$
\lim _{n \rightarrow \infty} \frac{\left\|J u_{n}-J y_{n}\right\|}{r_{n}}=0 .
$$

Observing that $u_{n}=T_{r_{n}} y_{n}$, one obtains

$$
f\left(u_{n}, y\right)+\frac{1}{r_{n}}\left\langle J u_{n}-J y, y-u_{n}\right\rangle \geq 0, \quad \forall y \in C .
$$


From (A2), one gets

$$
\begin{aligned}
\left\|y_{n}-u_{n}\right\| \frac{\left\|J u_{n}-J y_{n}\right\|}{r_{n}} & \geq \frac{1}{r_{n}}\left\langle J u_{n}-J y_{n}, y-u_{n}\right\rangle \\
& \leq-f\left(u_{n}, y\right) \\
& \leq f\left(y, u_{n}\right), \quad \forall y \in C .
\end{aligned}
$$

Taking $n \rightarrow \infty$ in above inequality, it follows from (A4) and (3.8) that

$$
f(y, p) \leq 0, \quad \forall y \in C .
$$

For all $0<t<1$ and $y \in C$, define $y_{t}=t y+(1-t) p$. Note that $y, p \in C$ and so one obtains $y_{t} \in C$, which yields that $f\left(y_{t}, p\right) \leq 0$. It follows from $(A 1)$ that

$$
0=f\left(y_{t}, y_{t}\right) \leq t f\left(y_{t}, y\right)+(1-t) f\left(y_{t}, p\right) \leq t f\left(y_{t}, y\right)
$$

that is,

$$
f\left(y_{t}, y\right) \geq 0 .
$$

Let $t \downarrow 0$. From (A3), we obtain $f(p, y) \geq 0$ for all $y \in C$, which imply that $p \in E P(f)$.

Finally, we show that $p \in V I(A, C)$. In fact, by (3.15), we have

$$
\left\|\Pi_{C}\left(J x_{n}-\eta A x_{n}\right)-x_{n}\right\| \rightarrow 0 \quad(n \rightarrow \infty) .
$$

Since $\lim _{n \rightarrow \infty} x_{n}=p$, we obtain

$$
\lim _{n \rightarrow \infty} z_{n}=p .
$$

By the continuity of the operator $J, A, \Pi_{C}$, we have

$$
\lim _{n \rightarrow \infty}\left\|\Pi_{C}\left(J x_{n}-\eta A x_{n}\right)-\Pi_{C}(J p-\eta A p)\right\|=0 .
$$

Note that

$$
\begin{aligned}
& \left.\| \Pi_{C}\left(J x_{n}-\eta A x_{n}\right)-p\right) \| \\
\leq & \left\|\Pi_{C}\left(J x_{n}-\eta A x_{n}\right)-x_{n}\right\|+\left\|x_{n}-p\right\| \rightarrow 0 \quad(n \rightarrow \infty) .
\end{aligned}
$$

Hence it follows from the uniqueness of the limit that $p=\Pi_{C}(J p-\eta A p)$. From Lemma 2.4, we have $p \in V I(A, C)$. Therefore, we have $p \in F$.

Step 5. $p=\Pi_{\digamma} J x_{0}$.

Since $p \in \digamma$, from the property (B3) of the operator $\Pi_{C}$, we have

$$
V\left(J \Pi_{\digamma} J x_{0}, p\right)+V\left(J x_{0}, \Pi_{\digamma} J x_{0}\right) \leq V\left(J x_{0}, p\right) .
$$

On the other hand, since $x_{n+1}=\Pi_{C_{n+1}} J x_{0}$ and $F \subset C_{n+1}$ for all $n \in \mathbb{N} \cup\{0\}$, it follows from the property $(\mathrm{B} 7)$ of the operator $\Pi_{C}$ that

$$
V\left(J x_{x+1}, \Pi_{\digamma} J x_{0}\right)+V\left(J x_{0}, x_{n+1}\right) \leq V\left(J x_{0}, \Pi_{\digamma} J x_{0}\right) .
$$

Furthermore, by the continuity of the operator $V$, we get

$$
\lim _{n \rightarrow \infty} V\left(J x_{0}, x_{n+1}\right)=V\left(J x_{0}, p\right)
$$


Combining (3.16), (3.17) with (3.18), we obtain

$$
V\left(J x_{0}, p\right)=V\left(J x_{0}, \Pi_{\digamma} J x_{0}\right) .
$$

Therefore, it follows from the uniqueness of $\Pi_{\digamma} J x_{0}$ that $p=\Pi_{\digamma} J x_{0}$. This completes the proof.

Remark 3.2. Theorem 3.1 improves Theorem 3.1 of Liu [11] in the following senses:

(1) The hemi-relatively nonexpansive mapping is more general than the relatively weak nonexpansive one in Liu [11].

(2) Our iterative algorithm (3.1) is completely different from the iterative algorithm of Theorem 3.1 of Liu [11].

(3) In contrast to Theorem 3.1 of Liu [11], our algorithm in Theorem 3.1 is related to three problems, that is, the fixed point, variational inequality and equilibrium problems.

When $\alpha_{n} \equiv 0$ in (3.1), we can obtain the new modified Mann iteration for the hemi-relatively nonexpansive mapping $T$, the variational inequality (1.1), the equilibrium problem (1.6) as follows:

Corollary 3.3. Let $E$ be a uniformly convex and uniformly smooth Banach space and $C$ be a nonempty closed convex subset of $E$. Let $f$ be a bifunction from $C \times C$ to $\mathbb{R}$ satisfying the conditions $\left(\mathrm{A}_{1}\right)-\left(\mathrm{A}_{4}\right)$. Assume that $A$ is a continuous operators of $C$ into $E^{*}$ satisfying the conditions (2.10) and (2.11), and $T: C \rightarrow C$ is a closed hemi-relatively nonexpansive mapping with $\digamma:=$ $F(T) \cap V I(A, C) \cap E P(f) \neq \emptyset$. For an arbitrary element $x_{0} \in C$, put $C_{0}=C$ and let $\left\{x_{n}\right\}$ be a sequence generated by the following iterative scheme:

$$
\left\{\begin{array}{l}
z_{n}=\Pi_{C}\left(J x_{n}-\eta A x_{n}\right), \\
y_{n}=\Pi_{C}\left(\alpha_{n} J x_{n}+\left(1-\alpha_{n}\right) J T z_{n}\right), \\
u_{n} \in C \quad \text { such that } \quad f\left(u_{n}, y\right)+\frac{1}{r_{n}}\left\langle J u_{n}-J y_{n}, y-u_{n}\right\rangle \geq 0, \quad \forall y \in C, \\
C_{n+1}=\left\{z \in C_{n}: \phi\left(z, u_{n}\right) \leq \phi\left(z, y_{n}\right) \leq \alpha_{n} \phi\left(z, x_{n}\right)+\left(1-\alpha_{n}\right) \phi\left(z, z_{n}\right)\right. \\
\left.\quad \leq \phi\left(z, x_{n}\right)\right\}, \\
\quad \prod_{n+1}=\Pi_{C_{n+1}} J x_{0}, \quad \forall n \geq 1,
\end{array}\right.
$$

where $\left\{\alpha_{n}\right\}$ is a sequence in $[0,1]$, and $\left\{\alpha_{n}\right\}$ and $\left\{r_{n}\right\}$ satisfies that $\liminf _{n \rightarrow \infty}$ $\alpha_{n}\left(1-\alpha_{n}\right)>0$ and $\left\{r_{n}\right\} \subset[a, \infty)$ for some $a>0$. Then the sequence $\left\{x_{n}\right\}$ converges strongly to a point $\Pi_{\digamma} J x_{0}$, where $\Pi_{\digamma}$ is the generalized projection from $C$ onto $F$.

If $T=I$ in the iteration algorithm of Corollary 3.3, it reduces to the new modified Mann iteration for the variational inequality (1.1) and the generalized equilibrium problem (1.6) as follows:

Corollary 3.4. Let $E$ be a uniformly convex and uniformly smooth Banach space and $C$ be a nonempty closed convex subset of $E$. Let $f$ be a bifunction 
from $C \times C$ to $\mathbb{R}$ satisfying the conditions $\left(\mathrm{A}_{1}\right)-\left(\mathrm{A}_{4}\right)$. Assume that $A$ is a continuous operators of $C$ into $E^{*}$ satisfying the conditions (2.10) and (2.11) with $\digamma:=V I(A, C) \cap E P(f) \neq \emptyset$. For an arbitrary element $x_{0} \in C$, put $C_{0}=C$ and let $\left\{x_{n}\right\}$ be a sequence generated by the following iterative scheme:

$$
\left\{\begin{array}{l}
y_{n}=\Pi_{C}\left(\alpha_{n} J x_{n}+\left(1-\alpha_{n}\right) J \Pi_{C}\left(J x_{n}-\eta A x_{n}\right)\right), \\
u_{n} \in C \quad \text { such that } \quad f\left(u_{n}, y\right)+\frac{1}{r_{n}}\left\langle J u_{n}-J y_{n}, y-u_{n}\right\rangle \geq 0, \quad \forall y \in C, \\
C_{n+1}=\left\{z \in C_{n}: \phi\left(z, u_{n}\right) \leq \phi\left(z, y_{n}\right) \leq \alpha_{n} \phi\left(z, x_{n}\right)+\left(1-\alpha_{n}\right) \phi\left(z, z_{n}\right)\right. \\
\left.\quad \leq \phi\left(z, x_{n}\right)\right\}, \\
x_{n+1}=\Pi_{C_{n+1}} J x_{0}, \quad \forall n \geq 1,
\end{array}\right.
$$

where $\left\{\alpha_{n}\right\}$ is a sequence in $[0,1]$ and $\left\{\alpha_{n}\right\}$ and $\left\{r_{n}\right\}$ satisfies that $\liminf _{n \rightarrow \infty}$ $\alpha_{n}\left(1-\alpha_{n}\right)>0$ and $\left\{r_{n}\right\} \subset[a, \infty)$ for some $a>0$. Then the sequence $\left\{x_{n}\right\}$ converges strongly to a point $\Pi_{\digamma} J x_{0}$, where $\Pi_{\digamma}$ is the generalized projection from $C$ onto $\digamma$.

Remark 3.5. See Remark 3.1 of Liu [11], Corollary 3.4 also does the corresponding promotions about $\mathrm{Li}[10]$ and Fan [7] as Liu [11]'s job.

If the mapping $A$ is a $\lambda$-inverse strongly monotone mapping in Corollary 3.3, then the following result can be also obtained by Corollary 3.3 and Theorem 3.1 .

Corollary 3.6. Let $E$ be a uniformly convex and uniformly smooth Banach space and $C$ be a nonempty closed convex subset of $E$. Let $f$ be a bifunction from $C \times C$ to $\mathbb{R}$ satisfying the conditions $\left(\mathrm{A}_{1}\right)-\left(\mathrm{A}_{4}\right)$. Assume that $A$ is a $\lambda$-inverse strongly monotone mapping of $C$ into $E^{*}$ satisfying the conditions (2.10) and (2.11) and $T: C \rightarrow C$ is a closed hemi-relatively nonexpansive mapping with $\digamma:=F(T) \cap V I(A, C) \cap E P(f) \neq \emptyset$. For an arbitrary element $x_{0} \in C$, put $C_{0}=C$ and let $\left\{x_{n}\right\}$ be a sequence generated by the following iterative scheme:

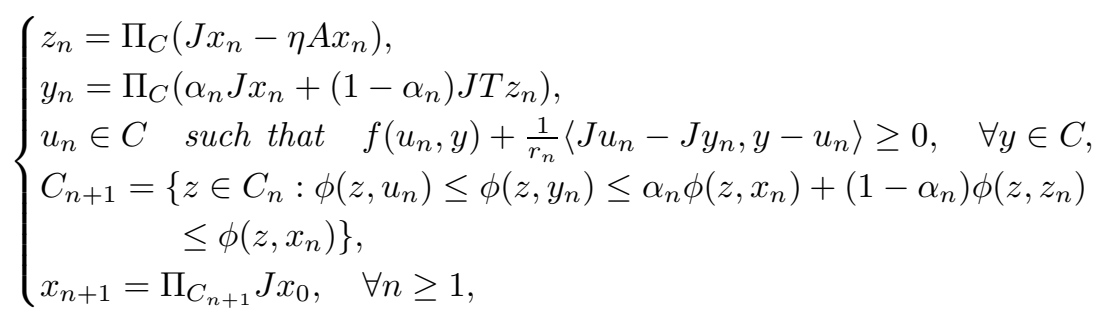

where $\left\{\alpha_{n}\right\}$ is a sequence in $[0,1]$ with $\liminf _{n \rightarrow \infty} \alpha_{n}\left(1-\alpha_{n}\right)>0$ and $\left\{r_{n}\right\} \subset$ $[a, \infty)$ for some $a>0$. Then the sequence $\left\{x_{n}\right\}$ converges strongly to a point $\Pi_{\digamma} J x_{0}$, where $\Pi_{\digamma}$ is the generalized projection from $C$ onto $\digamma$.

Proof. Since $A$ is $\lambda$-inverse strongly monotone, by (1.6), we have

$$
\|A x-A y\| \leq \frac{1}{\lambda}\|x-y\|
$$


for all $x, y \in C$, then it is Lipschitz continuous with constant $\frac{1}{\lambda}$. By Corollary 3.5 , we can directly obtain that the sequence $\left\{x_{n}\right\}$ converges strongly to a point $\Pi_{F} J x_{0}$.

Remark 3.7. Corollary 3.6 improves Theorem 3.1 of Takahashi and Toyoda [20] in the following senses:

(1) The hemi-relatively nonexpansive mapping is more general than a nonexpansive one in Takahashi and Toyoda [20].

(2) Our modified Mann iteration obtains strong convergence result about a $\lambda$-inverse strongly monotone mapping and a closed hemi-relatively nonexpansive mapping and generalized equilibrium problem (1.9) in a uniformly convex and uniformly smooth Banach space.

Acknowledgement. The authors thank the referee for the valuable comments and suggestions.

\section{References}

[1] Ya. Alber, Metric and generalized projection operators in Banach spaces: properties and applications, Theory and applications of nonlinear operators of accretive and monotone type, 1550, Lecture Notes in Pure and Appl. Math., 178, Dekker, New York, 1996.

[2] Ya. Alber and S. Reich, An iterative method for solving a class of nonlinear operator equations in Banach spaces, Panamer. Math. J. 4 (1994), no. 2, 39-54.

[3] E. Blum and W. Oettli, From optimization and variational inequalities to equilibrium problems, Math. Student 63 (1994), no. 1-4, 123-145.

[4] D. Butnariu, S. Reich, and A. J. Zaslavski, Weak convergence of orbits of nonlinear operators in reflexive Banach spaces, Numer. Funct. Anal. Optim. 24 (2003), no. 5-6, $489-508$.

[5] S. S. Chang, On Chidume's open questions and approximate solutions of multivalued strongly accretive mapping equations in Banach spaces, J. Math. Anal. Appl. 216 (1997), no. 1, 94-111.

[6] P. L. Combettes and S. A. Hirstoaga, Equilibrium programming in Hilbert spaces, J. Nonlinear Convex Anal. 6 (2005), no. 1, 117-136.

[7] J. Fan, A Mann type iterative scheme for variational inequalities in noncompact subsets of Banach spaces, J. Math. Anal. Appl. 337 (2008), no. 2, 1041-1047.

[8] S. Ishikawa, Fixed points by a new iteration method, Proc. Amer. Math. Soc. 44 (1974), $147-150$.

[9] S. Kamimura and W. Takahashi, Strong convergence of a proximal-type algorithm in a Banach space, SIAM J. Optim. 13 (2002), no. 3, 938-945.

[10] J. Li, On the existence of solutions of variational inequalities in Banach spaces, J. Math. Anal. Appl. 295 (2004), no. 1, 115-126.

[11] Y. Liu, Strong convergence theorems for variational inequalities and relatively weak nonexpansive mappings, J. Global Optim. 46 (2010), no. 3, 319-329.

[12] S. Matsushita and W. Takahashi, Weak and strong convergence theorems for relatively nonexpansive mappings in Banach spaces, Fixed Point Theory Appl. 2004 (2004), no. $1,37-47$.

[13] A. Moudafi, Second-order differential proximal methods for equilibrium problems, J. Inequal. Pure Appl. Math. 4 (2003), no. 1, 1-7.

[14] X. Qin, S. S. Chang, and Y. J. Cho, Iterative methods for generalized equilibrium problems and fixed point problems with applications, Nonlinear Anal. Real World Appl. 11 (2010), no. 4, 2963-2972. 
[15] X. Qin, Y. J. Cho, and S. M. Kang, Convergence theorems of common elements for equilibrium problems and fixed point problems in Banach spaces, J. Comput. Appl. Math. 225 (2009), no. 1, 20-30.

[16] X. Qin, S. Y. Cho, and S. M. Kang, Strong convergence of shrinking projection methods for quasi- $\phi$-nonexpansive mappings and equilibrium problems, J. Comput. Appl. Math. 234 (2010), no. 3, 750-760.

[17] S. Reich, Book Review: Geometry of Banach spaces, duality mappings and nonlinear problems, Bull. Amer. Math. Soc. (N.S.) 26 (1992), no. 2, 367-370.

[18] Y. Su, Z. Wang, and H. Xu, Strong convergence theorems for a common fixed point of two hemi-relatively nonexpansive mappings, Nonlinear Anal. 71 (2009), no. 11, 56165628.

[19] W. Takahashi, Nonlinear Functional Analysis, Yokohama-Publishers, 2000.

[20] W. Takahashi and M. Toyoda, Weak convergence theorems for nonexpansive mappings and monotone mappings, J. Optim. Theory Appl. 118 (2003), no. 2, 417-428.

[21] W. Takahashi and K. Zembayashi, Strong and weak convergence theorems for equilibrium problems and relatively nonexpansive mappings in Banach spaces, Nonlinear Anal. $\mathbf{7 0}$ (2009), no. 1, 45-57.

ZI-Ming WANG

DEPARTMENT OF Foundation

Shandong YingCAi UNIVERSity

JiNAN 250104, P. R. CHINA

E-mail address: wangziming1983@yahoo.com.cn

Mi KWANG Kang

Department of Mathematics

DONGEUI UNIVERSITY

Pusan 614-714, Korea

E-mail address: mee@deu.ac.kr

YeOL JE CHO

Department of Mathematics Education and the RINS

Gyeongsang National University

Chinju 660-701, KoreA

E-mail address: yjcho@gnu.ac.kr 